\title{
Nightingallery: theatrical framing and orchestration in participatory performance
}

\author{
Robyn Taylor • Guy Schofield • John Shearer • \\ Peter Wright $\cdot$ Pierre Boulanger $\cdot$ Patrick Olivier
}

Received: 17 May 2013/ Accepted: 27 November 2013

(C) Springer-Verlag London 2014

\begin{abstract}
The Nightingallery project encouraged participants to converse, sing, and perform with a musically responsive animatronic bird, playfully interacting with the character while members of the public could look on and observe. We used Nightingallery to frame an HCI investigation into how people would engage with one another when confronted with unfamiliar technologies in conspicuously public, social spaces. Structuring performances as improvisational street theatre, we styled our method of exhibiting the bird character. We cast ourselves in supporting roles as carnival barkers and minders of the bird, presenting him as if he were a fantastical creature in a fairground sideshow display, allowing him the agency to shape and maintain dialogues with participants, and positioning him as the focal character upon which the encounter was centred. We explored how the anthropomorphic nature
\end{abstract}

\footnotetext{
R. Taylor $(\bowtie) \cdot$ G. Schofield · J. Shearer · P. Wright ·

P. Olivier

Culture Lab, School of Computing Science,

Newcastle University, Newcastle upon Tyne, UK

e-mail: robyn.taylor@ncl.ac.uk

G. Schofield

e-mail: g.p.schofield@ncl.ac.uk

J. Shearer

e-mail: john.shearer@ncl.ac.uk

P. Wright

e-mail: p.c.wright@ncl.ac.uk

P. Olivier

e-mail: p.l.olivier@ncl.ac.uk

P. Boulanger

Advanced Man-Machine Interface Laboratory,

Department of Computing Science, University of Alberta,

Edmonton, Canada

e-mail: pierreb@ualberta.ca
}

of the bird itself, along with the cultural connotations associated with the carnival/sideshow tradition helped signpost and entice participants through the trajectory of their encounters with the exhibit. Situating ourselves as secondary characters within the narrative defining the performance/use context, our methods of mediation, observation, and evaluation were integrated into the performance frame. In this paper, we explore recent HCI theories in mixed reality performance to reflect upon how genre-based cultural connotations can be used to frame trajectories of experience, and how manipulation of roles and agency in participatory performance can facilitate $\mathrm{HCI}$ investigation of social encounters with playful technologies.

Keywords Digital arts · HCI - Participatory performance . Theatrical approaches - Practice-based research $\cdot$ Research in the wild · Interdisciplinary design

\section{Introduction}

Nightingallery is a digital performance installation where members of the public are invited to enter into a playful dialogue, singing and conversing with a musical bird. The focal point of the installation is a $60-\mathrm{cm}$ tall handcrafted animatronic, styled in a fashion evoking nineteenth century Victorian mechanical automata (see Fig. 1). The fantastical bird creature is accompanied by a team of live performers (see Fig. 2) who are also the designers and developers of the piece (Taylor. Schofield, and Shearer). The team present and showcase it to the public in a manner reminiscent of a carnival sideshow exhibition. As visitors approach and observe the bird character, their attention is drawn to the bird's melodic, chirping, birdsong calls. Visitors are able to use a microphone or telephone input 


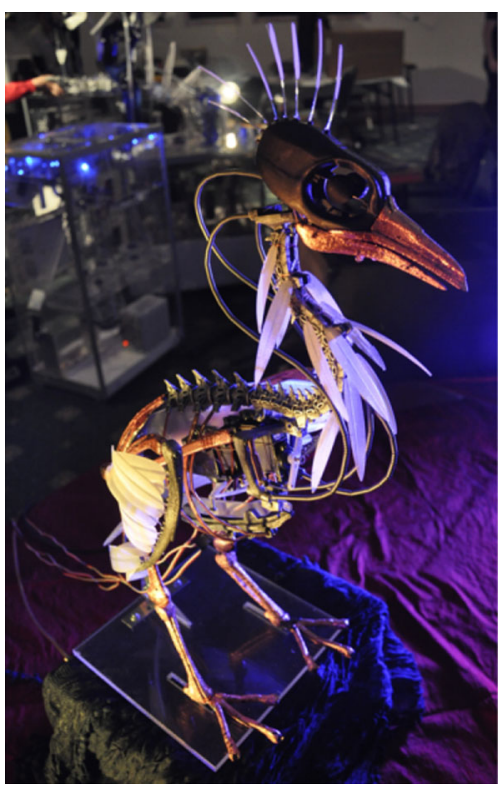

Fig. 1 Bird animatronic

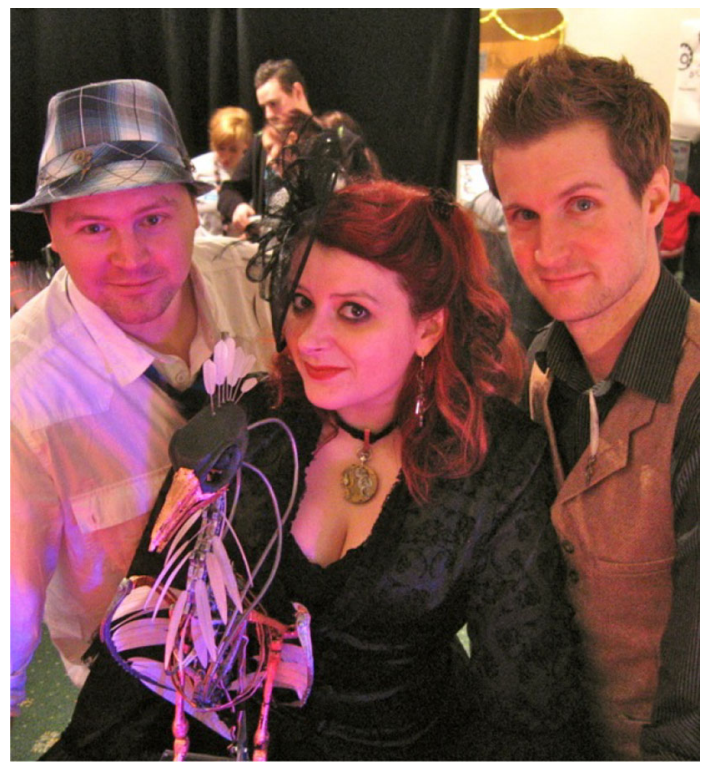

Fig. 2 Performance team

device (two alternative configurations developed for the system) in order to engage in verbal communication with the bird. This allows a dialogue to develop and evolve, with the bird character coaxing members of the public to converse and even sing with him. The bird encourages participants to experiment with their vocalizations, echoing and mimicking their voices and translating them into haunting, melodic birdsong. Through musical interaction with the bird character, members of the public are able to use the Nightingallery platform to experiment and explore improvisational music making and performance in public spaces. Nightingallery encourages permutations of public and private, spectatorial and participatory behaviour and was designed to facilitate social behaviours corresponding to the goals of our research: stimulating experience sharing amongst peers, and encouraging impromptu performance.

The Nightingallery project is part of a research practice that explores social behaviour in public performance spaces through the enactment and examination of interactive, performance-based artworks [28-30]. Our multidisciplinary team spans a variety of backgrounds, with members having professional experience in music and fine art in addition to training in HCI and design. By taking part as performers, we are able to intimately engage with participants and public in situ, communicating through dialogical, improvisational practices. This process of situated sense-making [13] unfolds through the intuitive and somatic [20] connections we develop with the audience and the piece.

In our previous use of interactive performance as an investigatory tool, we crafted experiences that, while participatory in nature, placed ourselves, the designers, firmly at the forefront of the action as musical performers. Using the format of a traditionally staged musical concert performance in dream.Medusa [28] and a busking scenario in humanaquarium [29], we engaged participants in shared experiences that had focused on exploring how interactive technologies could mediate participants' encounters and collaborations with live musicians (authors Taylor and Schofield.) The performer-centric format of these works proved particularly suitable for eliciting observational feedback surrounding the relationship between performers and audience-members-turned participants. However, during the course of enacting and analysing these performances, we were also able to observe numerous peripheral interactions between participants that took place during the performance. Many interesting social encounters were going on at the sidelines of the participants' primary engagement with the interactive performance, despite the fact that the performer-focused format of these works did not actively encourage or facilitate these kinds of interactions. In fact, in a set of post-performance interviews conducted as part of a study on dream.Medusa [28], participants expressed concern that inter-participant communication and interaction might be considered rude if it appeared to pull attention away from the live musical component of the interactive works.

When developing the Nightingallery project, we wanted to remove that performer-centric focus. We wanted to observe how participants collaborated and shared their experience amongst one another, and also to facilitate participants in creatively exploring improvisational possibilities-encouraging them to publically perform. Stepping back from the forefront of the installation, we cast 
ourselves in the role of carnival barkers and fantastical zookeepers (see Fig. 2), allowing and encouraging the bird animatronic to occupy centre stage in the performance. Our roles as performers were shifted to a strictly supporting capacity, functioning as what Benford and Giannachi term orchestrators of the experience [3], whose primary role was to facilitate participants' trajectories - the paths of discovery and exploration they followed as they encountered and experienced the work. Although much of Benford and Giannachi's work describes complex and structured mixed reality productions, in this paper, we describe how careful orchestration of trajectories can be used to enrich participant engagement during less formal, impromptu encounters of the sort characterized by Nightingallery. This paper illustrates how we deployed theatrical devices to provide cues and signposts for participants, smoothing what Benford and Giannachi caution are critical transition points that must be managed when designing and orchestrating a cohesive experience [3].

In this paper, we will describe how we accessed the cultural connotations of the carnival and steampunk genres and used the theatrical framing of the sideshow exhibition format to shape and signpost the trajectories of participants' encounters with the participatory performance platform. We will describe the compositional and aesthetic considerations that took place when designing and composing the work. We will also discuss how we used the theatrical framing of the Nightingallery performance to structure an HCI investigation exploring social behaviour in a variety of public spaces, including a BBC-sponsored concert and lecture series, a DIY craft and science exhibition and a major British rock music festival.

\section{HCI approaches to understanding the experience of participation in performance}

There is a growing body within the HCI research community that receives and values input from creative disciplines such as art and music. While such approaches may be found primarily on the edges of mainstream HCI research, they represent a trend towards the appreciation and awareness of multiple, dialogical interpretations of experience [22] and a recognition of the unique voice and perspective creative practitioners can lend to augment traditional HCI investigation [7]. A number of special interest groups (such as the CHI special interest groups in digital arts [6] and music [4]) and conferences (such as Creativity and Cognition) have emerged and developed, welcoming the contributions of interdisciplinary collaborators from the fine arts traditions.

By opening the study of human-computer interaction to encompass contributions gleaned from alternative disciplines, hybrid methodologies can be used to gain new perspectives on the relationships between people and technology. Wright et al. [32] propose a holistic, experience-centred design perspective, design-as-craft, which remains open to the influence of concepts and methodologies borrowed from alternative disciplines, such as that of art and craft practice. Höök et al. [9] suggest that art practice itself need not be considered separately from user study: the desire to provoke reaction to the artwork is inherently built into its design, and the manner in which this reaction is triggered and observed forms part of the message communicated by the work. Sheridan's work in Digital Live Art [23] combines aspects of HCI research with performance and installation traditions to explore social behaviour in playful spaces. Her performances create situated environments, in which risk-taking is permissible, and social norms are less restrictive. Sheridan et al. provide detailed discussion of how the truly playful and open scenario of the festival environment [24, 25] offers an authentic alternative to the artifice of the laboratory in terms of its ability to interrogate legitimately situated creative social experiences in shared public spaces.

Within the body of arts-based HCI research, there are many who look to performance as a provocative way to stimulate reflection and engagement in a manner that focuses on perception and experience [10]. Benford et al. [1-3], Reeves et al. [16, 17] and Sheridan et al. [23-26] have explored at length how the performance medium in particular has unique properties and conventions that make it well suited for exploring collaborative, public experience. This body of research examines the performance frame-the context within which the interactions, occurrences and behaviours that comprise the performance scenario take place [1] - and explores frameworks that can be used to describe and understand the roles of interactors in the participatory performance experience.

Sheridan et al. [26] identify what they term the tripartite interaction that takes place in an interactive performance scenario. The tripartite interaction model encompasses the roles of skilled performers, novice participants and the observing audience. This work explores how the interactive performance medium enables members of the public to develop from unwitting bystanders to fully witting audience members [23], even allowing them, if they so choose, to actively participate in the performance, transitioning from novice participants to skilled performers as they gain active and intentional control of the unfolding experience and the affordances of the performance interface [26].

Similarly, Benford and Giannachi explore a model of interaction that characterizes an interplay between spectators, performers and orchestrators [3], based on case studies of interactive works by Blast Theory and Thrill Laboratory [21]. In this conceptualization, the term 
"performers" is reserved for members of the public who play active, participatory roles in the execution of the works, while the term "orchestrators" is used to identify both the behind-the-scenes design team as well as the trained and rehearsed performers who play roles in the enactment of the performances. This manner of orchestration is exemplified in the work of Brendan Walker, who assumes the character of the Thrill Engineer when functioning as compère and showman during Thrill Laboratory events [3]. His performance as the Thrill Engineer helps orchestrate how the audience makes sense of the Thrill Laboratory, presenting it as the vision and masterwork of his charismatic persona. Under this representation, professional actors are considered to be part of the orchestration team-while they may be performing, their primary role is to facilitate the public's experience of the work. As this representation explicitly differentiates the confederate performers-members of the research team taking part in the project, whether in a backstage or performative capacity-from the visitors who encounter and take part in the installation, it allows an easy placement of Nightingallery's "designer/performer" within the orchestrator role. Our performative actions are intended to encourage participating novices to encounter the installation and take over the primary task of performance themselves, by developing and experiencing their own musical dialogues with the bird character.

Literature from performance studies stresses the importance of contextualization in performance. In Schechner's view, performance encompasses three phases: the gathering, the playing out of actions and the dispersing [18]. Taking an experience-centred approach to the evaluation of participatory performance and interactive installation art, Benford and Giannachi have specifically focused on the importance of crafting coherent trajectories through the phases of performance. Designers can signpost cues in order to guide visitors' engagement through the trajectory of an encounter with their creative works [3]. Benford and Giannachi explore how the Blast Theory projects envision the entire trajectory of participant experience with digital art installations, considering the duration of the proposed participant experience. Compositional decisions start from the beginning of the encounter, through the execution of the staged performance, and even, in some cases, into post-event reflection-through items secreted on the participant's person, intended for later discovery [3]. Thoughtful consideration during the composition phase of the Blast Theory projects ensures that while participants are free to navigate the interactive works as they see fit, their behaviours and outcomes are likely to roughly approximate the trajectory laid out by the composers-the canonical trajectory representing the idealized route that participants could follow as they engage with the artwork. Carefully placed suggestions and cues (such as those provided by the live interventions of confederate actors functioning as orchestrators) are intentionally made available in order to signpost, frame and guide participants' explorations as they navigate the world with the knowledge that as participants experiment and explore various aspects of the work, they will experience in actuality their own personalized participant trajectory through the encounter [3].

Cultural connotations also have significant impact upon the reception of a creative work. Explicitly exploring the use of culturally significant cues and formatting as a way to frame expectation, The Experiment Live, by Tennent, Martindale et al. [31] pays particular attention to contextualization, with all participating researchers and data collection methods carefully designed and disguised so as not to disrupt the coherence of the crafted experience. The audience is cued by the presentation format (in this case, a staged pseudo-scientific paranormal investigation) and by the use of culturally familiar signifiers (the medical props and monitoring paraphernalia evocative of the modern body-horror and parapsychology genres) in order to set the scene for a viscerally frightening participatory experience. The interactive narrative flows easily once the audience is primed for the scary story to begin. Situating the interaction within a familiar presentation style and signposting the narrative through the exposition of culturally connotative artefacts and references is key to the success of the project.

Nightingallery differs subtly from all these projects in that it was designed more in terms of street theatre than as a formally ticketed or invited performance. Participants were not briefed or introduced to the work, rather the piece was sited in locations where it could be encountered unexpectedly. We knew that the Nightingallery exhibit would likely be encountered as only one installation situated within a larger festival context. In order to create a cohesive experience, we explicitly made compositional choices which appropriated theatrical conventions and signifiers consistent with Nightingallery's chosen genre (steampunk/ dark carnival) in the hopes that through our own improvisational participation onsite, our orchestration of the simulated carnival scenario could provide a framing for the experience participants would have while interacting with our project. We hoped that by leveraging a familiar cultural context within which our creative content could be understood and appreciated, we could encourage people to play along with the conceit that the bird was really alivefacilitating a playful suspension of disbelief that would persist for the duration (however brief) of visitors' trajectories of interaction with the work. 


\section{Using theatrical form and genre to frame Nightingallery}

As mentioned in the outset of this discussion, our inspiration for the Nightingallery project grew out of observations and encounters we had when performing previous interactive art pieces through our process of practice-based research. As we designed the Nightingallery installation, we explicitly sought to choose a form of theatrical presentation that would encourage and promote the interparticipant social interactions we had observed taking place on the sidelines of our more performer-centric concertbased works. Instead of drawing attention and focus towards our own performances as we did in humanaquarium [29] and dream.Medusa [28], we wanted to create a performance platform in which we played a less dominant role, allowing participants greater freedom to interact expressively and communicate amongst one another. We also wanted to devise a work that could readily be presented within the context of a larger exhibition or fair, as we wanted to be able to adapt the performance to a variety of different presentation opportunities. Importantly, however, we wanted to ensure that the presentation format we selected provided us with a theatrically coherent framing within which visitors could encounter and experience the installation, even if it would generally be presented simultaneously alongside other works in a shared exhibition space, whatever its context.

In this section we discuss how aspects of theatrical convention were used as signposts to guide participants along the trajectories of their encounters with the Nightingallery project. Benford and Giannachi relate this type of participant experience to wayfaring [3], signposting cues to help individuals find their own way through a gradual process of experimentation and discovery, rather than following an explicitly defined route. When designing Nightingallery, we envisioned that alongside our active orchestration of encounters, theatrical conventions and signifiers would act as signposts guiding members of the public towards a trajectory in which they would (1) approach the installation (2) engage with the bird interface (3) develop their creative improvisations (either in the form of singing or conversation) and finally (4) conclude their encounter by disengaging with the installation and discussing their experiences with us and others around them.

\subsection{Framing through theatrical form}

We began to explore the potential of conceptualizing the Nightingallery experience in terms of a Victorian carnival sideshow. Visitors to our exhibit could be welcomed and greeted much as a carnival barker recruits fair-goers, with our function as orchestrators being to compete for and help retain the attention of passers-by, bringing them into our performance frame to present our carnivalesque oddity and attraction-the talking and singing animatronic bird. Choosing this form of presentation had several practical and conceptual benefits that immediately provided the work with foundational underpinnings, we felt would assist us in shaping interesting, productive public encounters with the piece:

- Positioning the work as one standalone element of a carnival sideshow allowed us to establish a theatrical narrative for an encounter that would begin and end at the allotted bounds of our self-contained portion of a shared exhibition space, providing us with a theatrical conceit that allowed us to capture participants' focus and attention for the duration of the interaction. Benford and Giannachi stress the importance of beginnings in managing participants' trajectories through an experience, citing admission, briefing and handing over of equipment as useful tools to smooth the transition [3]. In exploring a more impromptu form of encounter, many of these tools were not available to us. Attracting participants' attention through the set-up of the installation and our roles as carnival barkers was therefore critical as this was the only way for us to initiate the encounter.

- By casting ourselves in supporting, orchestrating roles, posing as the bird's assistants and minders or carnival barkers, we could intentionally and explicitly direct visitors' attentions towards the bird character through gesture and speech consistent with our theatrical characterization. Adopting this position would still allow us to orchestrate and facilitate participants' interactions if needed, without breaking character and damaging the theatrical integrity of the experience. Through this form of orchestration, we could intervene while still remaining in character, scaffolding participants' trajectories through the experience and guiding them towards fruitful engagement with the bird character.

- Presenting the bird character in the familiar casting of a sideshow oddity helped provide a context for the playful suspension of disbelief that participants would be required to engage in in order to enter into simulated dialogue with an obviously artificial mechanical bird. Benford and Giannachi discuss how the physical affordances of interfaces can be exploited to ease participants' transitions into taking up a particular role or beginning to interact with an artwork or interface. In the case of Nightingallery, the interaction space was entirely sonic and invisible; however, by supporting the conceit that the bird was a living character, we could signpost the participants as to the types of interactions possible.

Identifying the sideshow format as an appropriate theatrical form within which to present the work, we were 
then able to access the affordances of the presentation medium to signpost participants' trajectories through the encounter, cueing them using the cultural connotations of playfulness, curiosity and oddity borrowed from the nineteenth century carnival experience, and assisting them, if necessary, within the confines of the characterization we had composed for ourselves as secondary actors sharing the performance frame.

\subsection{Scene setting through genre}

Looking to the Victorian time period when conceptualizing Nightingallery allowed us to leverage many interesting cultural connotations when establishing the tone and feeling of the work. The genre of steampunk typically features a juxtaposition of modern and Victorian technological aesthetics [27]. Using elements of the steampunk genre in the visual design of the animated character, the stage setting, and the costuming, we could set the scene as visitors approached the installation, priming them to expect a certain type of experience. Referencing elements of late nineteenth century art forms in the Nightingallery project, we intended to evoke the interest in and curiosity towards technical spectacle commonly associated with the representation of the Victorian time period in popular culture, engaging with audiences through theatrical showmanship, technical ingenuity and technological novelty.

The late nineteenth and early twentieth centuries saw the birth of a huge number of technological innovations. Many of these inventions, involving new discoveries in optics, chemistry and engineering were used in sideshows and performances that were intended not only to entertain and educate the viewing public, but also to test-bed and raise awareness of new technologies [12]. The magic lantern shows which preceded the birth of cinema along with sideshows and presentations involving Tesla coils and other electrical innovations functioned in very similar ways to the new wave of modern day HCI research which uses art and performance strategies to present, discuss and explore technological innovations. Gunning, in writing about spectacular cinema, talks about the delicate state of technical appreciation and immersion in the work that cinemagoers inhabited during the first viewing of early films [8]. The sheer unfamiliarity and spectacle of moving imagery momentarily triggered a form of childlike credulity in an otherwise sophisticated audience who, of course, were fundamentally aware that the fantastical sights they were observing could certainly be explained by science and logic-however much they stimulated the imagination to conjure up explanations founded in miracle and mystique.
We hoped to leverage similar sensations of technical appreciation, curiosity, anticipation and surprise when a visitor was confronted with our automaton-a handcrafted mechanical bird apparently able to hold a responsive and believable English conversation with visitors to the installation. We hoped this would help foster a sense of enchantment and immersion that would encourage participants' active, prolonged engagement with the bird character, partially motivated, perhaps, by a desire to explore the technical possibilities of what the bird could and would do in response to their actions and contributions, as well as the clear level of craft skill involved in the creation of the physical artefact. In addition, the automaton as a cultural construct connotes a spectacle that is both fascinating and grotesque due to the undeniably imperfect replication of living motion and behaviour [14].

Much as how the visual presence of medical equipment sets the stage for The Experiment Live's horror scenario [31], the visual vocabulary provided by the animatronic bird character and the steampunk references present throughout the stage setting and theatrical costuming help establish participants' expectations from the outset of the Nightingallery experience. These visual vocabularies provide aesthetic cues as to the nature of what is about to unfold, specifically that visitors to Nightingallery should expect to be entertained in the manner reminiscent of a Victorian technical marvel, through the presentation of a technological artefact stimulating fascination and curiosity.

\section{The Nightingallery bird character}

In traditional performances such as concerts or plays, professional performers tend to fulfil a socially prominent function, initiating and maintaining participant engagement with the unfolding drama. Human performers augment themselves with elaborate costumes, makeup and studied personae in order to attract and maintain attention from audience members, or recruit and solicit attention from passersby. In the design process for Nightingallery, we speculated upon how our artificial character could draw upon similar resources, functioning as a sort of auto-pedagogical interface [15], to signpost and scaffold participants' trajectories through the Nightingallery experience. In particular the appearance and behaviour of the bird was designed to cue the participants into interacting with the interface in ways that loosely approximated the canonical trajectory of engagement, learning, improvisation and critical reflection that we had envisaged.

The bird character was chosen after discussing several ideas for the animatronic character persona. Approximating the figure of a bird when designing the automaton, we 
hoped to allow anthropomorphism of the installation, while avoiding uncomfortable sensations using a non-human character. In conceiving of a character that could plausibly engage visitors in spoken and sung verbal interaction, a bird seemed an obvious choice, bringing a ready-made set of affordances to potential encounters. By giving the bird the ability to vocally initiate interactions (through chirping and calling) and engage visitors in dialogues of increasing complexity, we hoped that the bird character would be able to maintain participants' focus throughout the encounter, enabling 'him' to hold the primary agency in the theatrical scene.

\subsection{Orchestrating interaction by framing the bird character as a "performer"}

During performances, authors Taylor, Schofield and Shearer played the roles of the bird's minders and attendants. We served as his assistants and maintained character throughout the duration of our encounters with visitors to the installation, conveying through our actions and words that the bird was the featured player and "star" of the performance scenario. We created costumes to wear during the performances, consistent with the aesthetic that formed the visual identity of the performance. Taylor combined Victorian corsetry with military accents, and Schofield and Shearer incorporated custom leatherwork and exposed clockwork mechanisms into their costuming (see Fig. 2). This resulted in us being easily recognizable as characters within the theatrical conceit of the Nightingallery performance; however, we were careful that our presence did not distract attention, which we wished to be focussed upon the bird.

While playing our roles as the bird's minders, we took a very theatrical approach to grooming and playing with him for deliberate effect (see Fig. 4), and in doing so established him as a legitimate performer who was the focus of the experience. This staging allowed us to reinforce the bird's character while also remaining available to mediate if the rapport between the bird and the participants was appearing to falter, and to reward participants' contributions through positive feedback and attentive encouragement (see Fig. 3).

We could occasionally sing or chat with the bird ourselves, intentionally exhibiting the range of its interactivity in order to attract attention to our installation or demonstrate the bird's vocal capabilities and singing prowess. However, once members of the public began to engage with the bird character, we consciously withdrew to the periphery of the interaction. Letting spectators assume the roles of performers, we intentionally moved further and further back in our capacity as orchestrators, intending that the focus of the experience be attenuated upon the

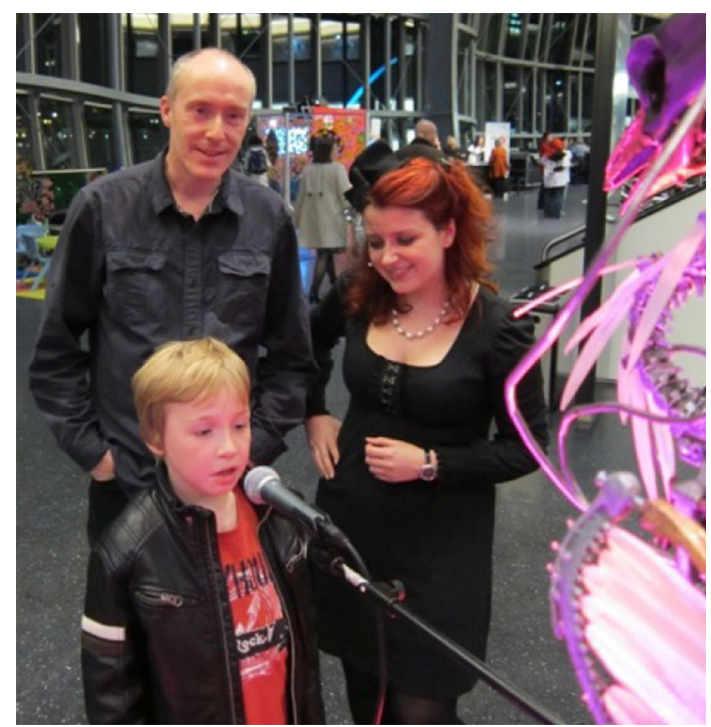

Fig. 3 Taylor encourages a child to sing and converse with the bird at the BBC Free Thinking Festival

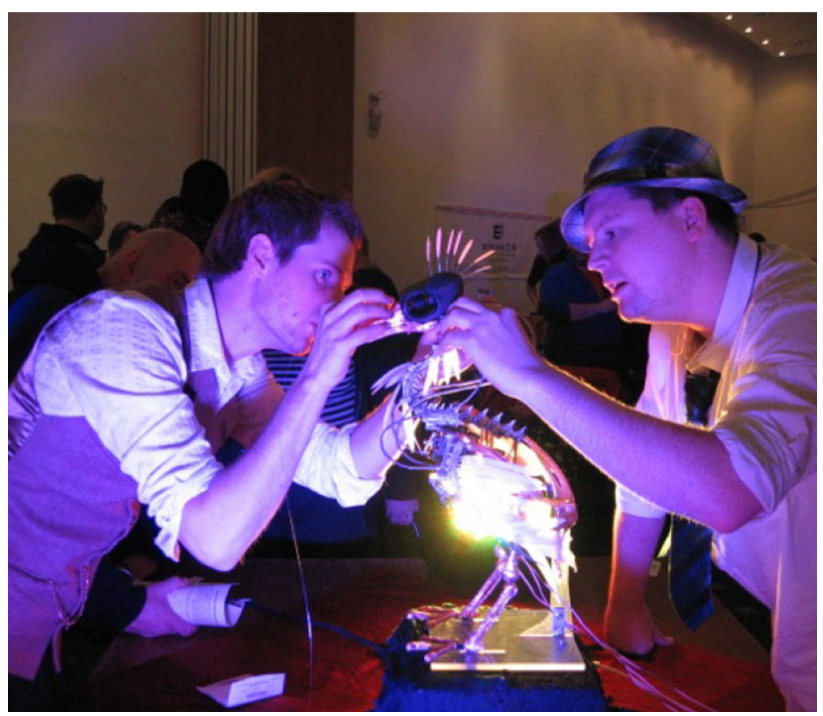

Fig. 4 Schofield and Shearer remain in character, even while attending to a repair of the bird's jaw mechanism at Maker Faire

developing dialogue between the visitors and the bird character.

Our team was careful to reinforce the bird's agency through chosen forms of speech. We deliberately cultivated the habit of referring to the bird as "him" rather than "it". We were intentionally consistent in this choice of pronouns and in our anthropomorphic assignment of motivations to the bird ("He can't hear you very well over the musicyou'll have to speak more loudly"). Consistently referring to the bird as if he were real, we encouraged participants to suspend disbelief and accept the bird as a player in the social setting and performance frame. 


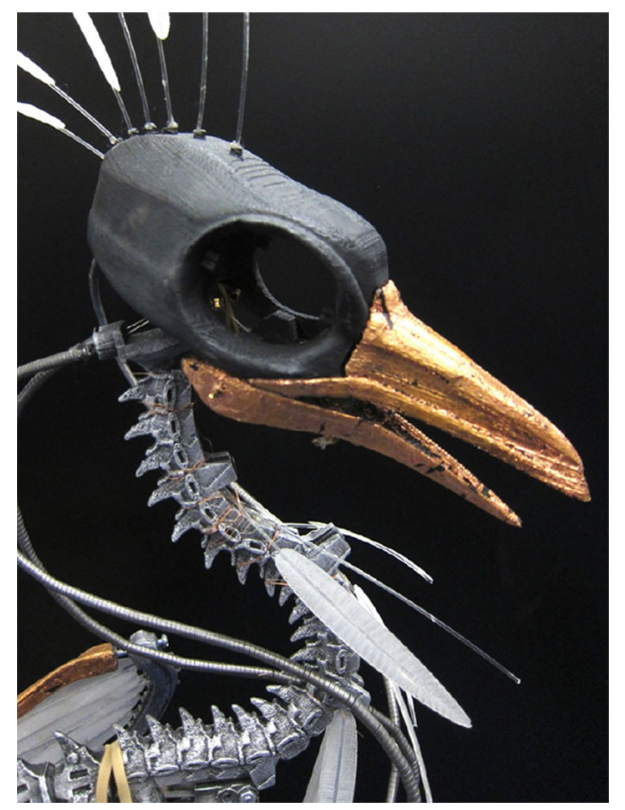

Fig. 5 Detail of the bird's head

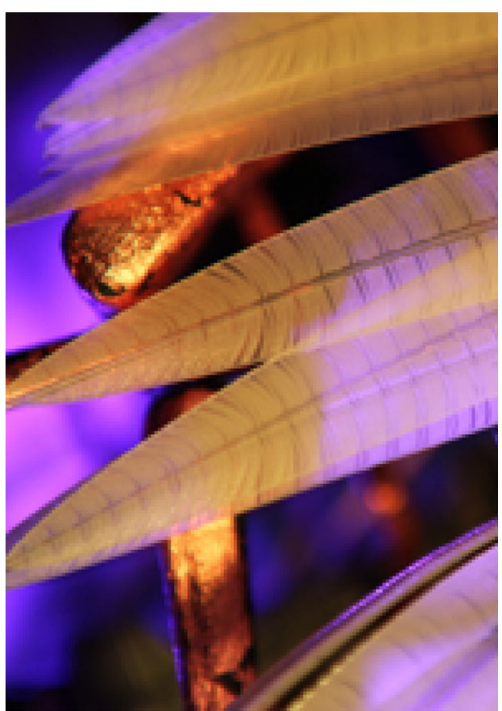

Fig. 6 Detail of the bird's feathers

\subsection{Crafting the bird's physical appearance and vocal behaviour}

Schofield, a trained sculptor and animator, created the bird's physical form using a combination of digital and traditional crafting techniques. The bird's design combined the visible mechanics of the animation hardware with references to Victorian taxidermy and stylized clockwork automata (Figs. 5, 6). The bird's physical behaviours responded to audio-based interaction through a MAX/ $\mathrm{MSP} / \mathrm{Jitter}$ interface, using an Arduino controller to drive movement.
The bird's voice was integral to the suspension of disbelief required to engage visitors with the installation narrative. For the sake of ascribing the bird a consistent character persona, we needed him to have a believably consistent vocal timbre, able to both speak a recognizable form of English that was "birdy" in nature, as well as chirp, twitter and sing in an appealing fashion.

The bird's vocalizations were synthesized based on human vocal input. By transposing human phrases and layering a number of vocal effects, we managed to establish a characterized speaking voice for the bird that remained relatively constant regardless of the gender or pitch of the spoken seed phrase. The end result could be understood as English, but had a character satisfyingly reminiscent of the croaky, cawing, harsh tonality of parrot or mynah bird speech.

In order to allow the bird to mimic and sing with participants, we used Max/MSP to equip the character with a fully synthesized singing voice that generated vocal content based on the frequency components present in the participants' vocalization. This resulted in a mimicking "birdsong" that shared an approximation of the same perceptual qualities as the input vocalization and created an instrument that was highly responsive to participant nuance. The bird's vocalizations were most dramatically tuneful when presented with harmonic, sung input. Harshness and choppiness found in spoken utterances (due to the presence of plosives and glottal stops found in speech) were reflected in the synthesized sound, making the bird chatter and chirp. When someone sang, however, his or her steady and strong vocal input would cause the bird to emit a rich, steady spectrum of musical sound. We hoped this would make risk-taking behaviour (singing) rewarding, and tempt participants to experiment with musical sounds in order to discover how the system would react, progressing towards creating their own improvisations.

\section{Interaction paradigms for the Nightingallery installation}

In the next sections, we discuss how our performancebased research practice informed the conceptualization and development of two interactive behaviour paradigms for the Nightingallery bird character. As previously discussed, we wanted to use the Nightingallery installation to explore how participants related to one another when interacting with unfamiliar technologies in public spaces. There were two particular social behaviours we wished to stimulate and explore.

The first was the sharing of the personal experience of the participant with his/her peers: we wanted to develop an 
interface that would stimulate participants to teach one another how to use the interface or recount their impressions of what had just transpired. The second was the use of the creative interface as a playful form of exhibitionism and impromptu performance in front of others. We wanted to see if we could encourage participants to intentionally "play to the audience" of those around them.

To investigate these social phenomena, we devised two distinct interaction paradigms for the installation, one that allowed participants to engage in complex dialogues with the bird via a telephone interface, and one which allowed participants to sing and perform with the bird using a conventional performance microphone.

As we discuss in more detail later, each paradigm was characterized by particular affordances that made it especially suitable for eliciting particular kinds of interactions. In addition, having two configurations of the system available made it possible for us to tailor the behaviour of the installation to the particular constraints of the realworld environments we found ourselves performing in during the course of touring and exhibiting the installation. Using the Nightingallery performance, we were able to explore how social interactions took place within a variety of public environments, including exhibitions ranging from as the crafts-and-DIY oriented Maker Faire, to a formal installation in the foyer of the BBC Free Thinking Festival (a concert and lecture series) to a muddy tent at Bestival, a popular British summer music festival.

\subsection{Interaction paradigm one: asymmetric telephone interface}

The first interaction strategy we devised was designed to explore how participants who interacted with playful technologies shared and communicated their experiences with those around them.

In order to do this, we looked for a way to create a significant distinction between the experience of the person participating and the experience of the onlookers who were observing, so that there could be unique content and information known only to the participant that she/he could then choose to communicate to others. We hoped this would stimulate interpersonal discussion, as the participant would have a private experience that she/he knew was not evident to those who remained on the public, observational side of the experience until it was explicitly disclosed.

We chose to implement an interaction scheme for the installation via an interface styled as a vintage telephone receiver, using the affordances of the telephone receiver device to facilitate an asymmetrically structured interaction paradigm. When a participant lifted the telephone receiver she/he could enter into a conversation with the bird through the telephone mouthpiece (see Figs. 7, 8). The affordance

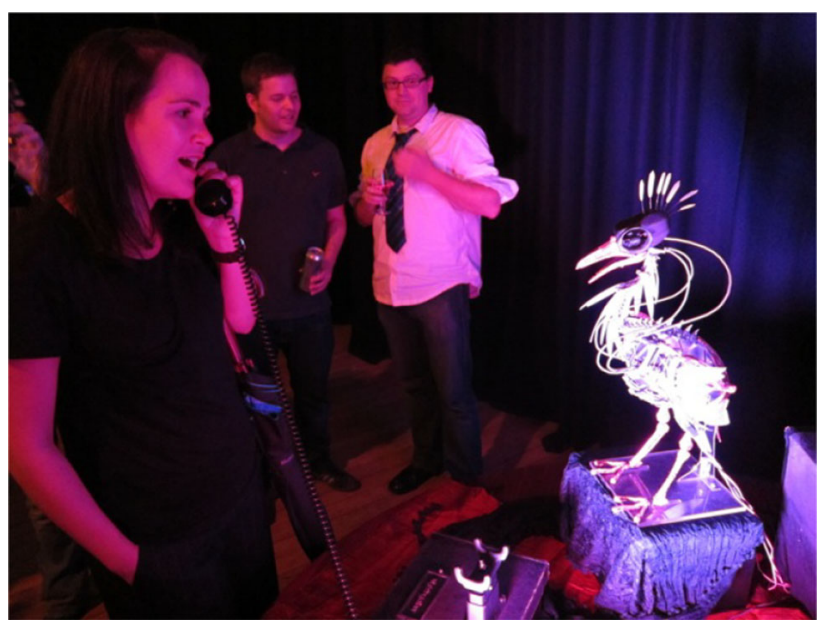

Fig. 7 Visitor has a phone conversation at JAM46 (an art and performance event)

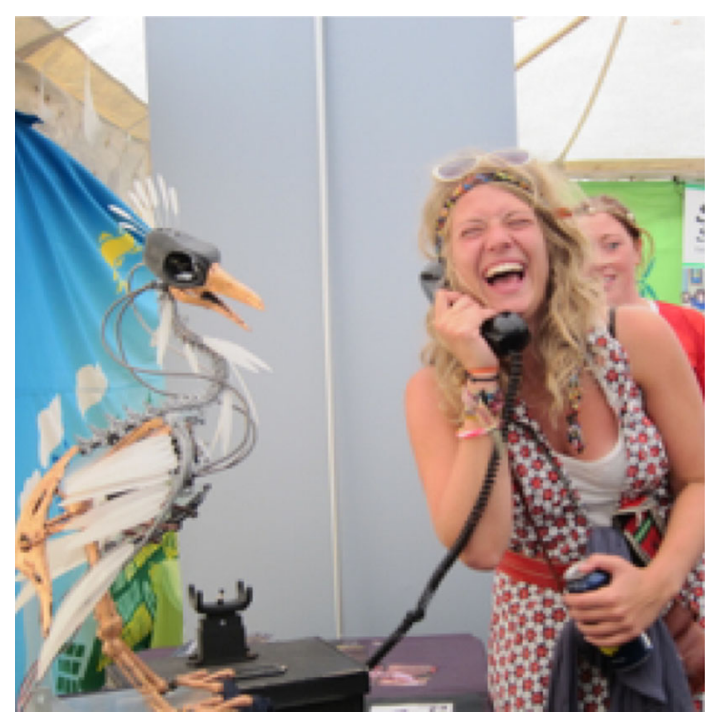

Fig. 8 Visitor has a phone conversation at the Bestival festival

of the telephone receiver enabled the participant to speak quietly if she/he so wished, out of earshot of the crowd. The bird's responses were transmitted as recognizable English through the earpiece of the phone, forming a private channel of content available only to the participant directly interacting via the telephone.

To the onlooking spectators who were not privy to the communications heard only through the earpiece, the bird's voice sounded only like unintelligible birdsong (played through a loudspeaker concealed in the base of the bird's platform.) The observing audience could see the bird's beak moving and hear the cadences of the birdsong, but no intelligible words could be discerned. This method of feedback was intended to provide entertainment for the viewing public (via the bird's melodic and dynamic 
birdsong responses) but only the person holding the handset would be able to understand the meaning of the conversation. The content of the conversation could only become known if the participant chose to tell people what was said, giving the participant the ability to share the details of what was heard through the phone interface, or to keep the information to him or herself.

By allowing individuals to decide how much to reveal about their interaction, we gave them partial control over what Reeves et al. [17] refer to as the "spectator experience", whereby public interaction is defined in terms of manipulations - the observable physical actions undertaken by the participant upon the interface (in the case of Nightingallery, speaking into the telephone receiver) and effects - the results of the manipulations (the bird's vocal responses). Using the phone interface in Nightingallery, people could choose to enact secretive interactions by speaking quietly into the phone (the manipulation) and refraining from communicating what was said in response (the effect), or, instead, could choose to conduct more expressive interactions by speaking with the intent to be overheard and observed. In any case, the asymmetric design would stimulate a sense of suspense for onlooking spectators, as one could only experience what was happening on the receiver portion of telephone interface through taking a turn at participating firsthand.

\subsubsection{Scripting the telephone conversations}

To initiate interactions, the telephone would ring, enticing a passer-by to pick up the receiver. The person on the phone would then hear a bird-like voice through the earpiece of the telephone, repeating "Hello? Hello?" until she/he vocally replied, initiating a conversation which would terminate when the phone was replaced on its hook.

We modelled the bird's behaviour after the popular culture representation of the type of pet bird known for vocal mimicry-a parrot or a mynah bird. When scripting the bird's vocalizations, we discussed how we would expect an entertaining bird character to behave and watched numerous examples of people playing with mimicking birds online. Much of the entertainment factor provided by a talking bird appeared to relate to the potential anthropomorphism of an animal capable of making sounds recognizable as English, but evidencing logic very definitively not that of a human. We wanted to stimulate a sense of anticipation and humour in participants who engaged with the Nightingallery installation, intending to develop maddeningly repetitive vocal behaviours for the bird, yet allowing him to maintain participants' interest through unpredictability, surreality and comedy.

As participants spoke into the telephone, the system recorded their verbal utterances for future playback. These recorded phrases were used to augment a pre-recorded memory bank of scripted bird phrases. The bird's pre-recorded phrase library contained a variety of phrases that either:

- Furthered the conversation by soliciting participant response (such as "why are you doing this?" or "tell me a story?"). By questioning the participants in this manner the bird to lead the narrative of the encounter, much in the way a human performer might thus reinforcing the bird's agency.

- Evoked the cultural context that installation was crafted to reflect, referencing ominous quotes from Victorian literature (such as "beware the jubjub bird" or "fair is foul and foul is fair"). Choosing disconcerting phrases from familiar literary sources helped us establish the mood of the encounter and was in keeping with the aesthetic of the bird's dishevelled, steampunk appearance. This reinforcement of genre was intended to help prolong participant engagement by signposting the nature of the interaction we hoped they would experience (engaging in an uncanny, eerie dialogue.)

By recording participants' phrases into the bird's memory bank, the library of responses became more customized and varied as the conversation went on. Bizarre conversations would evolve if participants played along and responded to a conversational entity whose cultural familiarity as a mimicking, parrot type of creature allowed them to excuse his fundamental absurdity. The dialogue, while absurd, could be interpreted as acceptably believable when considered within the context of a human/bird narrative that we had established through our theatrical framing and aesthetic references to Victorian automata, technological artifice and carnival oddities.

\subsection{Interaction paradigm two: a conventional microphone interface}

In addition to exploring how people shared experiences amongst social groups, we were also interested in using the Nightingallery installation to investigate how we could reduce participant inhibitions and motivate them to perform more creative, performative actions in collaboration with the bird character. During our initial Nightingallery exhibitions using the asymmetric telephone interface, we had observed a number of participants who, by providing dynamic phrases and sounds for the bird to sing and repeat, chose to perform for the public through the medium of the bird.

The cadence of the birdsong that was broadcast over the loudspeaker followed the cadence of the spoken phrases, meaning that if the bird repeated a participant's particularly emphatic phrase, the observing audience would be able to recognize the vocal pattern in the repetition. Several 
participants were seen to exploit this feature, saying or singing things in exaggeratedly pitched voices to make their friends laugh. Observing these social behaviours reinforced to us that the bird animatronic was in fact tempting some participants to use it for creative means.

We chose to design a second alternate configuration for the system, implementing an interaction scheme that we hoped would even more readily facilitate impromptu performance. We hoped that the physical layout of this configuration would be better suited to attracting and sustaining the attentions of participants and passersby in noisy, boisterous social environments.

In this configuration, we replaced the telephone interface with a conventional microphone (see Figs. 9, 10). The microphone was positioned so that the participant could easily see the bird at eye level and interact with him in full view of his/her social group. This layout intentionally referenced the trappings and affordances of a conventional stage upon which the participant could perform, which we hoped would help cue visitors to the installation that performative behaviour was acceptable and welcome.

In this interaction paradigm, the private channel of communication was removed and all efforts focused upon encouraging participants to publicly perform with the bird by making him respond to and imitate their voices. In this implementation, we chose to simplify the bird's behaviour greatly, making him purely a mimic. When a participant spoke or sang a phrase into the microphone, the bird would respond (after a short fixed interval of roughly half a second) with a stylized "birdsong" echo of the participant's utterance. We hoped that the repetitive nature and

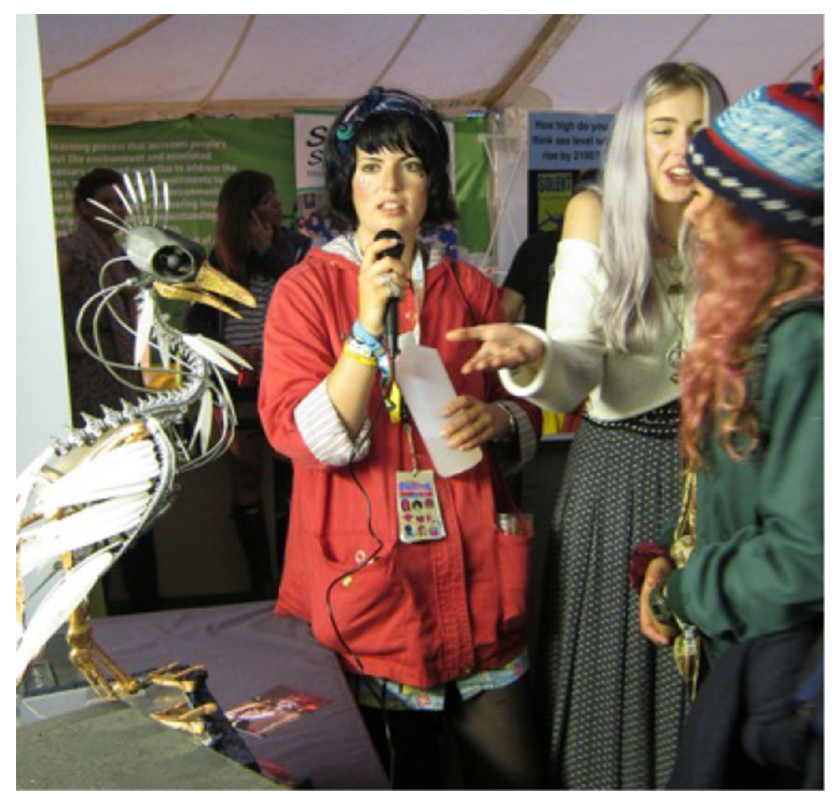

Fig. 9 Participants at Bestival sing to the bird using the microphone interface

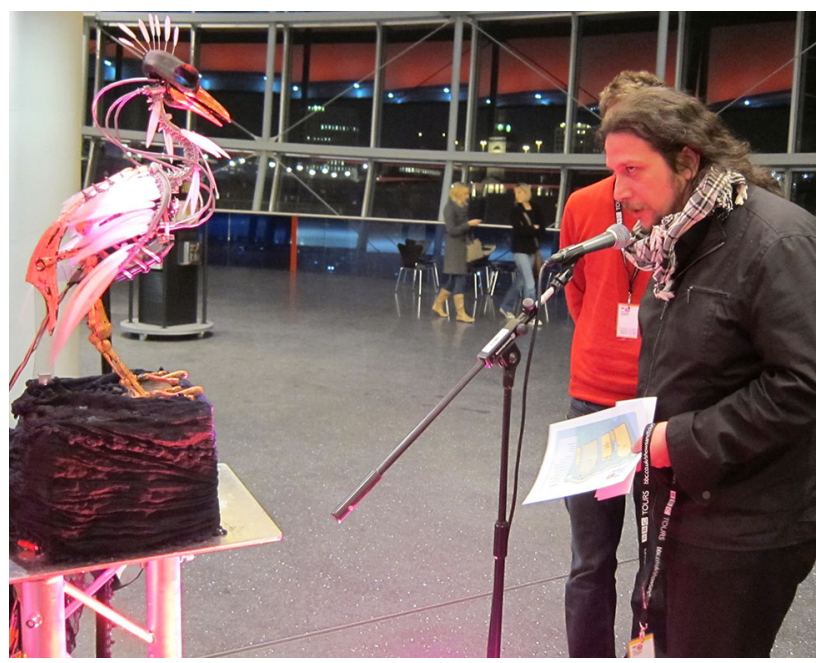

Fig. 10 Participants at the BBC Free Thinking Festival sing to the bird

predictable timing of the bird's behaviour would focus participants on testing how they could control the range and extent of the bird's vocal mechanism, tempting them to "perform" through the bird character. Allowing participants to directly control the audible content of the bird's mimicking reply meant that they could feel confident that they could predict what was going to happen after they spoke or sung. As described later in this document, exploitation of this knowledge led to participants' ability to develop more sophisticated musical constructs in collaboration with the bird character.

\section{Findings and discussion}

In order to engage with as wide a range of participants as possible, we scheduled a number of public exhibitions of Nightingallery in very different settings. The installation was debuted at the Maker Faire UK exhibition in 2011. Maker Faire UK is a large public craft and DIY fair held annually in Newcastle upon Tyne incorporating around 300 demonstrations and attended by over 5,000 people. A popular family-friendly event, Maker Faire attracts a crosssection of the local community as well as visitors from across the UK interested in DIY culture. Nightingallery was presented in a large open space alongside a varied selection of other work, ranging from homemade electronic instruments to robotics to handicrafts.

Subsequently, the installation was shown at BBC Radio Three's Free Thinking Festival at the Sage Concert Hall in Gateshead, a debate, lecture and performance festival aimed at a literary audience featuring contemporary thinkers and speakers such as Germaine Greer. In contrast, the installation was later exhibited at Bestival (a large UK 
outdoor music festival combining thematic installation art with concert appearances by high-profile rock bands such as headliners The Cure) attended by over 50,000. Additionally Nightingallery was shown at many smaller exhibitions often connected to media and technical arts festivals or consortia. These additional venues included Dorkbot at the International Centre For Life, the British HCI conference and JAM46, an art and performance event held at Culture Lab Newcastle. During each presentation, we were careful to maintain the theatrical integrity of Nightingallery as a self-contained experience, always framing it as a sideshow rather than a technical demo.

Having the two system configurations at our disposal (the telephone-based system which facilitated more complex, private dialogues between the participant and the bird, and the microphone system which allowed the participant to undertake more straightforward and publically conspicuous interactions), we were able to easily switch between the two interaction strategies, sometimes to target the research to particular concerns and sometimes to respond to practical considerations brought about by the characteristics of the environments we found the installation located within.

In terms of supporting our research questions, for example, at Maker Faire, the large number of families and children in attendance allowed us to investigate how closeknit family groups shared their experiences with the unfamiliar technology. To directly explore this, we used the telephone interface, as it permitted us to construct a situation where one family member had a personal experience to share. In contrast, we often made use of the microphone interface at the Bestival to explore whether we could channel the energies of festival-goers and encourage them to perform for their peers. The uninhibited, creatively oriented atmosphere of the Bestival environment made the use of this configuration particularly rewarding, as visitors were particularly inclined to use Nightingallery to animatedly sing and perform with their friends. The BBC Free Thinking Festival provided us with yet another scenario, as not only did many of the visitors have classical music training, but the installation was sited in a quiet foyer with excellent acoustics. In this situation, the microphone configuration allowed us to explore whether more complex musical interactions would take place.

As an example of configuring the system in response to practical considerations related to the exhibition environment, we found that while the telephone interface worked well in spacious environments where people could hear and concentrate well, it was sometimes tricky to use in fastpaced, noisy settings. Participants occasionally found it hard to follow the content of complex conversations if many distracting things were happening around them. At Bestival, for example, the high level of noise and activity experienced during the boisterous evening hours meant that the microphone interface was, for practical reasons, a better choice of configuration.

In any case, having both configurations at our disposal allowed us to reconfigure the system in response to what transpired during an exhibition. In practice, we found we often switched between them several times over the course of a multiple-day exhibition, in order to see how we could yield interesting, diverse and fruitful results.

While the respective affordances of microphone and telephone seem to suggest inherent properties regarding public vs private behaviour, in this section, we will go on to discuss how we observed participants shaping what Reeves terms the spectator experience [17] of their public interactions with Nightingallery, constructing their own boundaries of public versus private behaviour through how they chose to visibly engage with the interfaces that allowed them to communicate with the bird character. Dalsgaard and Hansen's theory of performing perception [5] explains how participants who engage with a publicly situated system such as the Nightingallery installation are simultaneously aware of (1) their own interactions with the system, (2) their perception of the relationship between themselves, the system, and their surroundings and (3) their conscious knowledge that they are in fact performing their actions in view of others who observe. Using the Nightingallery installation as a platform for HCI exploration, we were able to observe how members of the public performed their perception of the experience in numerous different ways, their own behaviours reconfiguring expectations of public and private boundaries between themselves, the bird character, the spectating audience and us as orchestrators also present within the performance frame.

We focused our study on the two themes that formed the research goals for the project: how participants communicated and shared their personal experiences with others, and how participants used the Nightingallery platform to perform and music-make. We will elaborate upon each of these themes in this remaining section, and discuss how the use of performance and theatricality in the way that Nightingallery was presented helped shape how participants encountered and experienced it "in-the-wild".

\subsection{Sharing private experiences with others}

Dalsgaard and Hansen [5] also explore how mobile phone users habitually draw upon performative strategies to demarcate a private boundary within a public space. This conceptualization of mobile phone use positions the user simultaneously as operator, spectator and performer, interacting with the device, consciously aware of how they appear to others and performatively defining their desired privacy (or lack thereof) through their actions and 
demeanour. Using Nightingallery's asymmetrical telephone interface, we were able to observe how participants chose to share (or conceal) their private individual experience with friends, family or peers.

Participants did this in a variety of ways. Some participants chose to explicitly recount the contents of the phone call. This either happened during the call (a participant might echo a phrase the bird said to them on the phone, allowing onlookers to understand the conversation as it was happening) or after they had terminated the phone call and finished interacting with the bird. The level of detail included in participants' recounting of the conversation would vary. Some participants would echo or paraphrase the specifics of dialogue (e.g.: "It said 'hello' back!") while others would provide more high-level commentary about the call (e.g.: "It kind of repeats what I say, but in a 'birdy' way." or "It's completely bonkers!").

Some participants chose a more indirect method of communicating their private experience-a method which was explicitly theatrical-illustrating how the Nightingallery platform allowed performing to feel like a natural way of communicating and sharing. Instead of recounting what had happened on the phone, some participants chose to exaggerate their end of the conversation for the purpose of conveying the content of the phone call to others. This method of communication is what Schechner terms showing-doing - explicitly performing, illustrating, and underlining what is being experienced or done [19]. Through showing-doing, some participants were able to creatively convey an interesting narrative solely through the phrases they contributed to their end of the conversation. By following the contextual and dialogical clues contained in each comment the participant spoke into the telephone mouthpiece, onlookers could approximately infer the other end of the conversation ("No, I won't tell you a story! Stop asking me that"). One particularly memorable performance had a participant animatedly ordering a pizza over the telephone, expressing pretend frustration as if the mimicking bird was a particularly incompetent pizza shop staff member who was not cooperating in taking the order. This method of communication deliberately subverted the conventionally private affordance of the telephone handset interface; increasing the performative qualities of the phone conversations in order to share and communicate in creative, expressive ways.

Another form of communication that we occasionally observed alerted us to a potential avenue for creativity that we had not originally envisioned when designing the system. We observed participants inventing and sharing behaviours that could not possibly have occurred given the constraints of the interface. On one occasion a child repeatedly insisted to her parents that when she asked the bird-specific questions (like favourite colour, or name) the bird responded with content that we, as the programmers, knew was not contained in the bird's phrase bank. She made up a very detailed description of the conversation she insisted that she had had which we did not choose to contradict! The child's parents left the installation quite impressed with the "intelligence" of the talking bird-due to a complete fabrication believably conveyed by their child's recounting. This intrigued us, as it illustrated how the existence of the private channel of experience gave the child the ability to bluff — crafting her own narrative which she chose to recount to her family, using the bird as a character in her own storytelling process.

After talking to the bird, many participants encouraged their friends and family to discover the telephone experience by having a conversation of their own. Enthusiastic participants would often physically pass the phone receiver to others, encouraging them to speak into the mouthpiece. Some participants motivated their friends to try the telephone by deliberately refusing to recount their experience, insisting that to find out what happened on the phone, their friends and family must have a conversation for themselves.

Many of these teaching and assisting interactions, straightforward as they were, carried a strong performative connotation. By persuading others to "talk to the bird" and by agreeing to play along with the conceit that one had just engaged in a worthwhile dialogue with the creature ostensibly on the other end of the phone line, the participating audience member was most certainly performing and even transitioning somewhat to a role approximating orchestration, convincing others to participate and immerse themselves more deeply in the frame of the developing performance. Participants' willingness to recruit and assist their friends and peers to use the system also indicated a reassuring level of commitment to and approval of the installation: if someone was willing to coerce a friend into experiencing the installation-if they had that impulse to share-it shows us that they found their own experience worthwhile enough to invest their own personal credibility by recommending it to others.

Configuring the installation to use the asymmetric telephone interface allowed us an easy way to examine how people learned the interaction scheme and then pass on this learning to their peers through the aforementioned variety of direct and indirect means. While the design of the bird character had been intended to engage one participant at a time, this experience sharing helped participants to transfer their knowledge, easing the learning process for their peers. Predictably much of this occurred through direct recounting and intentional teaching, however, surprisingly, participants also subverted the conventional interaction scheme of the telephone dialogues by communicating their learned experience publically, through performing for their 
peers, or even crafting their own invented narratives (such as in the case of the pizza delivery bird or the bluffing child who made up a complex story about an impossible dialogue). By performing their perception [5] of how other audience members were interpreting their hidden interactions with the system, participants were able to shape the performance, contributing their own creative content to spectators' experiences of the work.

\subsection{Performing and music making}

The second set of observations we collected surrounded how participants were able to use the Nightingallery installation to explicitly perform and engage in musicmaking activities. In our experiences, complex music making was most directly observed when the system was configured using the microphone-based system, as no subversion of the interface was required in order for it to be commonly understood that the person in front of the microphone could sing and perform with and alongside the bird character.

We knew at the outset that persuading participants to sing in public would be a challenging task, as social norms do not traditionally reward inexperienced singers for attracting attention by singing in public spaces. The aesthetic and functional aspects of the installation were designed to encourage participants to overcome the perceived cultural barrier that made the act of singing in public somewhat intimidating. We also tried to cultivate a positive and welcoming environment through our own participation in the shared narrative, using dialogue ("He really likes it when you sing to him!") and attentive body language to urge and encourage participants to sing.

Participants rapidly discovered that even speaking or singing quietly would still cause the bird to respond with an audible musical birdsong corresponding to what they had said or sung. Shy participants could thereby allow the bird to do the performing rather than risk being heard singing themselves, often cupping their hands over the microphone so that their own vocal contributions were inaudible to others sharing the space.

Alternatively, groups of people could participate together. Often the more confident singers would gravitate towards the microphone, while their more hesitant peers could sing from a distance. In this way, the entire group could share in the appropriation of the experience. Even participants who were unwilling to sing themselves could hold their mobile phones up to the microphone, playing MP3 s for the bird to translate into birdsong. This subversion of the installation allowed participants to take part in the experience in a creative way that we had not envisioned.

We observed evidence suggesting that the aesthetic of the bird's musical capabilities was sufficiently engaging that participants were tempted to experiment with increasingly complex vocalizations, overcoming shyness in order to explore the interaction space of the bird's musical responses. The most striking example of this behaviour saw a small girl's experimental interaction progress from random noises and utterances through to simple songs, culminating in her crowd-stopping rendition of a complex coloratura run from Mozart's Queen of the Night's Vengeance Aria. Her progression in confidence from hesitant explorer to crowd-pleasing virtuoso performer took place over the course of several visits to the installation during an exhibition day and remains the most exhilarating example of Nightingallery's ability to encourage members of the public to enjoy sharing their talents in a reassuring, welcoming space.

While the above illustrated an impressive example of a participant gaining the confidence to engage in a series of progressively more remarkable vocal performances, we were also able to observe numerous participants who developed complex and unique music-making strategies as they explored the interaction paradigm. Some participants were able to devise and refine increasingly nuanced ways of interacting with the bird responses, building interesting and sophisticated musical structures through experimentation and practice. After becoming familiar with the behaviour of Nightingallery's interaction system, in particular becoming attuned to the timing of the delay between participant input and system response, these participants incorporated rhythmic aspects to their interactions. Call-and-response was a popular style for these types of interactions, whereby participants would perform simple songs with the bird, timing the rhythm of their phrasing to the rhythm of the bird's replies. More sophisticated still was the attempt to use the bird to sing cyclical "rounds". An professional choral singer in attendance at the Free Thinking Festival managed to overlay her live vocals over the bird's vocal playback in order to build up a multi-voiced song sung in the manner of a traditional multiply-voiced round.

Presenting Nightingallery within the contexts of cultural events like the Bestival festival or the BBC Free Thinking Festival often illustrated how the spatiotemporal aspects of the environment played a large role in facilitating how participants would choose to interact with the musical interface. Sometimes, qualities of the performance venue itself would stimulate participants to make specific creative choices in their improvisational interactions with the bird.

If external music sources were present in the environment, participants often attempted to use the bird to sing along or jam with the music playing in the space. A particularly memorable incident happened at Bestival when a dedicated group of participants determined how to manipulate the timing and duration of their vocalizations in order to allow the bird to sing along with remarkable 
accuracy to a Bob Marley track playing over a nearby loudspeaker. Generally, when interacting with Nightingallery at music festivals, participants were very likely to sing excerpts from artists featured on the concert line-up. It was interesting to see participants using the bird in this manner, relating to and entertaining their peers by referencing artists and songs which were currently relevant within the festival context.

Through the process of encouraging and observing singers interacting with the system, we remained attuned to the fact that performance is a form of experience sharing. The performers were generally very acutely aware that they were being observed. In some cases, this intimidated them, and in other cases, it encouraged them to seek further positive attention and social reinforcement. Singing well (or singing comically badly), choosing situationally relevant songs (such as the Bestival patrons singing $\mathrm{Bob}$ Marley or hits of headliners The Cure) or performing crowd-pleasingly impressive arias (like the small child's Queen of the Night performance), allowed participants to solicit positive social feedback from those around them, both strangers and friends. Positive reactions from those around them encouraged participants to continue making the bird sing and continue developing their confidence and expertise with the creative interface.

As Dalsgaard and Hansen point out [5], a performer in this situation could be seen as simultaneously performing as well as maintaining a spectatorial awareness of how she/ he is being perceived by others nearby. If no engaged audience was present, our performance team was available onsite to fulfil the role of performing spectator [5], visibly providing supportive social feedback and positive reinforcement for the participants' music-making activities, orchestrating and facilitating this aspect of the experience from our position on the periphery of the established performance frame. Remaining in character, we would occasionally comment on participants' performances, with statements such as "He loves whistling..." If participants seemed to have run out of ideas about how to interact, we could offer hints and encouragements such as "Nobody's sung to him yet today..." or suggestions based on what previous participants had done. Consistent with other research investigating social behaviour in creative, playful environments [11], the co-presence of others (whether members of the public or ourselves as orchestrators filling the spectatorial role) helped each individual make sense of the boundaries defining the situation by watching how those around them interacted and behaved, helping them judge what actions would be well received. By positioning ourselves as orchestrators within the space, we allowed participants to take cues from our behaviour and social feedback, while finding and defining their own trajectories through the work.

\subsection{Theatrically framing the experience}

As previously mentioned, we were able to present the work in a variety of social contexts. In some of these, the intentionally theatrical nature of our presentation style fits naturally with the exhibition context (such as was the case during art or music festivals like Bestival) whereas others (particularly the scientific conferences or technology expositions like Maker Faire) required us to maintain a more concerted effort in order to persuade visitors to engage in kind with fanciful speech and theatrical affect. Regardless, we felt that consciously conceiving of our exhibition of the work as a performance rather than a demonstration had conceptual merits, not the least of which was that by adopting a theatrical, creative demeanour we were effectively modelling the playfulness and improvisational willingness we hoped to elicit and support.

The use of the sideshow context as a framing device for our work was chosen specifically to allow us to respond to a widely differing range of exhibition contexts, enabling us to consistently and cohesively establish and maintain a creative space within which we could invite participants to experiment and explore. Using performative and visual cues to signpost the experience in terms of a sideshow exhibition, we were able to communicate a demarcation of our own theatrical space, both physically within the larger exhibition context and creatively, in terms of the narrative trajectory participants engaged in when interacting with the work. This was particularly useful when we exhibited the project as part of large events like Maker Faire and Bestival, where neighbouring stalls were likely to contain presentations as distractingly diverse as scientific experiments or hands-on crafting demonstrations. At the Free Thinking Festival, the quieter, less chaotic environment permitted longer engagements to take place and generally required less intervention and support on our part; however, the sideshow format was still useful in establishing and shaping participants' expectations, serving as a coherent framing that allowed them to rapidly make sense of the type of experience that was about to unfold. In each case, the carnival/sideshow framing also allowed us to directly orchestrate the initiation of the interaction through our characterization as carnival barkers, recruiting and greeting visitors in character and guiding them if needed through the trajectory of their own personal encounters with the work.

As part of our characterization, we intentionally feigned a respectful deference to the bird character, with the intention of establishing him as a persona with presence, agency and social dominance within the scenario. This was often rewarded when we observed participants' responses to his vocal exhortations to interact and sing. 
His imperious demands were often obeyed. Even when they were not, participants' refusals were often accompanied by language ("No I won't tell you a story!") which demonstrated willingness to engage with the theatrical scenario-if not a willingness to comply with the bird's demands, at least to comply with the conceit that he was real. As we became more practiced with the format, we found that straight-faced absurdity often produced heightened response: most memorably, we horrified an intoxicated Bestival-goer, remarking offhandedly that when the bird was "off-duty" during the night-times, he often entertained himself by malevolently prowling between the rows of tents.

Our dialogue and the bird's requests explicitly instructed participants as to what type of interactions they were invited to explore, and the interaction apparatus (telephone and microphone) provided easily accessible signposts demarcating the affordances of the interface and cueing participants' engagement with the trajectory of the narrative space. Talking on a telephone has a socially understood suggestion of one-on-one dialogue, with the narrative of a phone call having a structured beginning (lifting the receiver) and end (when the receiver is replaced). Similarly, a microphone has a conventional usage that carries a performative connotation. Using these familiar devices as cues helped us to elicit the music making and experience sharing behaviours we had hoped to stimulate through our practice-based research.

By maintaining a consistently theatrical approach throughout, we were able to orchestrate the trajectory that visitors took through the encounter, shaping how the narrative progressed, beginning with their initial approach, during their interactions with the character, and even as they brought the encounter to a close. This strategy also allowed us to smoothly integrate into the encounter questions and comments that often arose after a visitor had played with the installation, as their curiosity turned to how the installation worked and what the project was about. These questions, which in another scenario might have forced a jarring disconnect, could instead be woven into the theatrical experience. The sideshow form if anything encouraged visitors to look "behind the curtain", transitioning between immersing themselves in the roleplay aspects of the interaction and engaging critically with the craft and technology involved in its creation. This strategy approximated the function of the souvenirs and replay interfaces suggested by Benford and Giannachi, encouraging reflection, discussion and the sharing of memories [3].

By building this detailed theatrical performance around the bird character, we created the set of conditions that were particularly conducive to explore the social interactions we had set out to elicit and observe.

\section{Conclusions}

The Nightingallery project enabled groups of people to engage in shared interactions with a digital artefact in public spaces, allowing a range of social phenomena to be explored. The interactive installation featured an animatronic automaton, effectively drawing visitors in while the unfolding theatrical narrative guided them through the increasingly complex interaction paradigms that we wished to explore.

Reflecting on our experiences and unpacking why Nightingallery was successful in encouraging participants to engage and make music with the animatronic bird character, it is clear that there are numerous aspects of the design that came together to create a space where improvisational, playful behaviour was permissible and even encouraged. By performing physically alongside visitors, using our performances to orchestrate and support visitors' participation rather than to provide the central aesthetic content of the work, we provided participants with a "safe space" to step into and engage in improvisational play and music making. The use of the sideshow format as a framing device, and the cultural connotations conveyed by referencing the steampunk genre helped participants make sense of a narrative encounter, establishing expectations about the nature of the experience from the outset. In addition, the interface mechanisms (the telephone and microphone) were easily understood and laden with contextual associations that suggested how they could be used. Together, all of these design choices contributed to the crafting of an experience that scaffolded and encouraged communication, collaborative play and improvisational performance.

In keeping with Benford and Giannachi's conceptualization of an experience as a trajectory from initial encounter all the way through to post-encounter appropriation [3], when structuring our investigation of social behaviour in public spaces we carefully considered how each detail of our design would signpost participants' trajectories as they interacted with Nightingallery, implicitly provoking the social behaviours we wanted to observe. Benford and Giannachi demonstrated how considering participant experience in terms of a trajectory could be used to craft and coordinate elaborate mixed reality productions. We have shown how the same strategies can effectively be used to orchestrate impromptu encounters in less structured settings, signposting participant trajectories via implicit cues built into the design that are framed by an appropriate genre and theatrical form.

Our investigation was intended to shed light on two social phenomena: performance and the sharing of experiences amongst participants; however, we found that these two features were often inextricably linked. As was evident from the interactions we described, communications 
amongst peers were oftentimes conveyed using performative methods, and additionally, many instances of creative performance were spurred by a desire to share a collective experience amongst friends. Participants would share their experiences in the process of engaging with the interface, often through performance. Bluffing, role play and storytelling were often not only pure play but also used simultaneously as pedagogical tools, leading others into the performance.

Presenting Nightingallery as a performance work allowed us to investigate these particular aspects of social interaction, but importantly, it also allowed us more generally to explore the effects of using an unusual interaction scheme which exploited non-traditional affordances: the sung voice and an audio-kinetic "display" (the animatronic bird). The way that this research was structured, in terms of cueing through genre and theatrical form, could be usefully applied to other HCI projects, especially those investigating how users might be encouraged to engage with unfamiliar interfaces. Using theatre as a framing device, Nightingallery illustrates how a stylized presentation format can help researchers' structure participants' engagement with scenarios under investigation. In our case, we were exploring unusual interaction schemes applied to non-traditional, playful interfaces; however, it could be easy to extrapolate the practice, using theatrical framing and creative narratives to increase participant engagement with any number of human-computer interacting investigations.

By taking active roles in the participatory experience, we were able to engage in practice-based HCI research, interrogating the performance experience while taking part in it. Theatrical framing allowed us to create an environment where whimsical, playful improvisation was socially acceptable, and even encouraged, a process which was vital in scaffolding the types of interaction we wished to observe. This environment enabled audience members to approach, engage and ultimately lead the performance, negotiating the terms of their engagement between themselves, each other and the installation. Audience members became the primary performers as we ourselves transitioned towards a less conspicuous placement in the performance frame, orchestrating the experience from within and exploring how theatrically framing encounters between people and technology could encourage and facilitate sharing and creative play in public space.

Acknowledgments Figures 1, 2 by Cassim Ladha. Figure 3 by Qasim Chaudhry. Figure 6 by Ko-Le Chen.

\section{References}

1. Benford S, Crabtree A, Reeves S, Sheridan J, Dix A, Flintham M, Drozd A (2006) The frame of the game: blurring the boundary between fiction and reality in mobile experiences. In Proceedings of the SIGCHI conference on human factors in computing systems, ACM, New York, pp 427-436

2. Benford S, Fraser M, Reynard G, Koleva B, Drozd A (2002) Staging and evaluating public performances as an approach to CVE research. In Proceedings of the 4th international conference on Collaborative virtual environments, CVE'02, pp 80-87

3. Benford S, Giannachi G (2011) Performing mixed reality. The MIT Press, Cambridge

4. Bevilacqua F, Fels S, Jensenius AR, Lyons MJ, Schnell N, Tanaka A (2013) SIG NIME: music, technology, and humancomputer interaction. In CHI EA'13

5. Dalsgaard P, Hansen LK (2008) Performing perception: staging aesthetics of interaction. ToCHi: transactions of computerhuman interaction, $15: 3-4,13.11-13.33$

6. England D, Fantauzzacoffin J, Schiphorst T, Latulipe C, Candy L (2013) Digital art: challenging perspectives. In: CHI EA'13

7. Gaver W (2007) Cultural commentators: non-native interpretations as resources for polyphonic assessment. Int J Hum Comput Stud 65(4):292-305

8. Gunning T (1989) An aesthetic of astonishment: early film and the incredulous spectator. Art Text 34:31-45

9. Höök K, Sengers P, Andersson G (2003) Sense and sensibility: evaluation and interactive art. In:Proc CHI'03, pp 241-248

10. Jacucci C, Jacucci G, Wagner I, Psik T (2005) A manifesto for the performative development of ubiquitous media. In Proceedings of the 4th decennial conference on Critical computing: between sense and sensibility (CC '05), NY, pp 19-28

11. Jacucci J, Spagnolli A, Chalambalakis A, Morrison A, Liikkanen L, Roveda S, Bertoncini M (2009) Bodily Explorations in space: social experience of a multimodal art installation. In Proceedings of INTERACT '09, 62-75

12. Lightman B (2007) Victorian popularizers of science: designing nature for new audiences. University of Chicago Press, Chicago

13. McCarthy J, Wright P (2004) Technology as experience. The MIT Press, Cambridge

14. Munster A (2006) Materializing new media: embodiment in information aesthetics (interfaces: studies in visual culture), Dartmouth College Press, Hanover, New Hampshire

15. Penny S, Smith J, Sengers P, Bernhardt A, Schulte J (2001) Traces: embodied immersive interaction with semi-autonomous avatars. Convergence 7(2):47

16. Reeves S (2011) Designing interfaces in public settings: understanding the role of the spectator in human-computer interaction (1st ed.). Springer

17. Reeves S, Benford S, O'Malley C, Fraser M (2005) Designing the spectator experience. In Proc CHI'05, pp 741-750

18. Schechner R (1988) Performance theory. Routledge, New York

19. Schechner R (2002) Performance studies: an introduction. Routledge, New York

20. Schiphorst T (2011) Self-evidence: applying somatic connoisseurship to experience design. In CHI EA'11, pp 145-160

21. Schnädelbach H, Egglestone SR, Reeves S, Benford S, Walker B, Wright M (2008) Performing thrill: designing telemetry systems and spectator interfaces for amusement rides. In Proceedings of CHI '08, pp 1167-1176

22. Sengers P, Gaver B (2006) Staying open to interpretation: engaging multiple meanings in design and evaluation. In Proceedings of the 6th conference on designing interactive systems, DIS'06, pp 99-108, New York

23. Sheridan JG (2006) Digital live art: mediating wittingness in playful arenas. PhD Thesis. Computing Department, Lancaster University, Lancaster

24. Sheridan JG, Bryan-Kinns N (2008) Designing for performative tangible interaction. Int J Arts Technol 1(3/4):288-308 
25. Sheridan J, Bryan-Kinns N, Reeves S, Marshall J, Lane G (2011) Graffito: crowd-based performative interaction at festivals. In Proc. CHI'11 Extended Abstracts, pp 1129-1134

26. Sheridan J, Dix A, Lock S, Bayliss A (2004) Understanding interaction in ubiquitous guerrilla performances in playful arenas. In Proc HCI pp 3-18, Springer

27. Tanenbaum J, Tanenbaum K, Wakkary R (2012) Steampunk as design fiction. In Proc. CHI '12, pp 1583-1592

28. Taylor R, Boulanger P, Olivier P, Wallace J (2009) Exploring participatory performance to inform the design of collaborative public interfaces. CHI Extended Abs 3721-3726

29. Taylor R, Schofield G, Shearer J, Boulanger P, Wallace J, Wright P, Olivier P (2011) Designing from within: humanaquarium. In
Proceedings of the 2011 annual conference on Human factors in computing systems (CHI '11). ACM, New York, pp 1855-1864

30. Taylor R, Schofield G, Shearer J, Boulanger P, Wallace J, Wright $\mathrm{P}$, Olivier P (2011) Composing for the interactive medium. In the Proceedings of the 13th International Conference on Virtual Reality, Laval

31. Tennent P, Martindale S, Marshall J, Reeves S, Walker B, Harter P (2012) Performing the experiment live, Proceedings of ACM CHI Workshop on Exploring CHI's Relationship with Liveness, Austin

32. Wright P, Blythe M, McCarthy J (2006) User experience and the idea of design in HCI. Interactive Systems, 12th (LNCS):1-14 